but not the BCL2 or BCL6 loci. There was no $M Y C / I G H$ reciprocal translocation by dual colour dual fusion probe.

Her serum levels of albumin, globulin, total protein, creatinine, calcium, immunoglobulin (Ig)A, IgG, and $\operatorname{IgM}$ were all within normal ranges. Serum protein electrophoresis was negative for any paraprotein. Magnetic resonance imaging (MRI) scans of the lower back 9 months prior to the oral biopsy showed lumbar radiculopathy, without evidence of any osteolytic bone lesions. Furthermore, the tumour cells expressed CD19, which is mostly negative in plasmacytoma. All these findings excluded the differential diagnosis of plasma cell myeloma or plasmablastic myeloma. The patient achieved complete remission after reduction of the immunosuppressants to half of the dosages (methotrexate to $5 \mathrm{mg}$ per week and prednisolone to $5 \mathrm{mg}$ per day) in June 2018, without chemotherapy or radiation therapy. She remained free of disease or recurrence for 13 months as of June 2019 with the reduced dosages.

EBVMCU was first described by Dojcinov et al. in 2010, and so far there have been less than 100 reported cases in the literature. ${ }^{4}$ In a recent review, Prieto-Torres et al. broadened the spectrum of EBVMCU and described some unusual features, such as cases presenting as multiple lesions in a single anatomical location, monomorphic large cell morphology, angioinvasive pattern, PDL1 expression in large B-cells, and EBER expression in the adjacent epithelial cells. ${ }^{5}$ In our case, we found many CD20 negative, lambda light chain-restricted plasmacytic/plasmablastic cells with clonal immunoglobulin receptor gene rearrangement and MYC rearrangement, which has not been described in EBVMCU yet. This finding has broadened the histopathological spectrum of EBVMCU and raised a challenging differential diagnosis with PBL. There are two morphological patterns of PBL, one with monomorphic plasmablastic cells and the other with more mature plasma cells. Nevertheless, a polymorphic infiltrate with large lymphocytes, eosinophils, plasma cells, and histiocytes is characteristic for EBVMCU as in our case, but not in PBL. The large cells in EBVMCU usually express B-cell markers (CD20, CD79A, PAX5, BOB1, and OCT2), CD30, IRF4/MUM-1, and LMP1. In contrast, the tumour cells of PBL are negative for CD20 and PAX5, and rarely positive for LMP1. About one-third of EBVMCU cases are clonal for immunoglobulin receptor gene or T-cell receptor gene rearrangement, which may lead to a misdiagnosis of B- or T-cell lymphoma. ${ }^{6}$

In conclusion, the current case broadens the histological spectrum of EBVMCU, as rare cases like ours can show plasmacytic/plasmablastic immunophenotype with clonal Bcell receptor gene rearrangement and $M Y C$ rearrangement. Clinical and pathological correlation is essential as these cases need to be differentiated from PBL or plasmablastic myeloma or EBV positive plasmacytoma.

Conflicts of interest and sources of funding: The authors state that there are no conflicts of interest to disclose.

\footnotetext{
Bo-Jung Chen ${ }^{1}$, Wei-Fan Chiang ${ }^{2,3}$, Tsang-Shan Chen ${ }^{4}$, Hsu-Ju Kao ${ }^{5}$, Shih-Sung Chuang,

${ }^{1}$ Department of Pathology, Shuang-Ho Hospital, Taipei Medical University, New Taipei City, Taiwan; ${ }^{2}$ Department of Dentistry, Chi-Mei Medical Center, Liouying, Taiwan;
}

${ }^{3}$ Department of Dentistry, School of Dentistry, National Yang Ming University, Taipei, Taiwan; ${ }^{4}$ Department of Neurology, Sin-Lau Christian Hospital, Tainan, Taiwan; ${ }^{5}$ Department of Hepato-Gastroenterology, Chi-Mei Medical Center, Liouying, Taiwan; ${ }^{6}$ Department of Pathology, ChiMei Medical Center, Tainan, Taiwan; ${ }^{7}$ Department of Pathology, School of Medicine, National Taiwan University and Taipei Medical University, Taipei, Taiwan

Contact Shih-Sung Chuang, MD.

E-mail: cmh5301@mail.chimei.org.tw

1. Bunn B, van Heerden W. EBV-positive mucocutaneous ulcer of the oral cavity associated with HIV/AIDS. Oral Surg Oral Med Oral Pathol Oral Radiol 2015; 120: 725-32.

2. Hart M, Thakral B, Yohe S, et al. EBV-positive mucocutaneous ulcer in organ transplant recipients: a localized indolent posttransplant lymphoproliferative disorder. Am J Surg Pathol 2014; 38: 1522-9.

3. Daroontum T, Kohno K, Eladl AE, et al. Comparison of Epstein-Barr virus-positive mucocutaneous ulcer associated with treated lymphoma or methotrexate in Japan. Histopathology 2018; 72: 1115-27.

4. Valera A, Balague O, Colomo L, et al. IG/MYC rearrangements are the main cytogenetic alteration in plasmablastic lymphomas. Am J Surg Pathol 2010; 34: 1686-94.

5. Prieto-Torres L, Erana I, Gil-Redondo R, et al. The spectrum of EBVpositive mucocutaneous ulcer: a study of 9 cases. Am J Surg Pathol 2019; 43: 201-10.

6. Dojcinov SD, Venkataraman G, Raffeld M, et al. EBV positive mucocutaneous ulcer-a study of 26 cases associated with various sources of immunosuppression. Am J Surg Pathol 2010; 34: 405-17.

DOI: https://doi.org/10.1016/j.pathol.2019.07.003

\section{Phenytoin-associated necrotising lymphadenitis mimicking Kikuchi disease}

Sir,

Histiocytic necrotising lymphadenitis, also known as Kikuchi disease, usually affects young adults of Asian descent who present typically with fever and painless, unilateral cervical lymphadenopathy. ${ }^{1}$ Most cases follow a benign, self-limited course with spontaneous remission within a few months. The aetiology remains unclear. Both autoimmune and infectious aetiologies, especially viral infection, have been suggested, ${ }^{1}$ but an association with phenytoin administration has not been reported. Pathologically, Kikuchi disease is characterised by a mixed population of plasmacytoid dendritic cells, histiocytes, $\mathrm{T}$ cells and immunoblasts with many cells undergoing apoptosis. ${ }^{2}$ Three morphological patterns have been described, and a temporal relationship has been suggested from proliferative phase to necrotic and then xanthomatous phase. $^{2}$ However, the full morphological spectrum of Kikuchi disease is found only rarely in a single biopsy specimen. Thus, the well-known differential diagnosis may include lymphadenopathy in systemic lupus erythematosus (SLE) and infectious lymphadenitis. ${ }^{3}$ Here, we report a case of phenytoin (Dilantin)-induced necrotising lymphadenitis which mimicked Kikuchi disease.

A 23-year-old Taiwanese man presented with convulsive status epilepticus characterised by episodic focal seizures with intermittent secondary generalisation in the past 12 months. He carried the HLA-B*1502 allele and took 
levetiracetam and lacosamide as baseline anti-epileptic medication. ${ }^{4}$ The status epilepticus was controlled initially with valproic acid and levetiracetam; medication was changed to phenytoin on admission to avoid the risk of liver failure. Three days later, intermittent low-grade fever developed which was followed by spiking fever $\left(39.2^{\circ} \mathrm{C}\right)$ one week later. In addition, generalised maculopapules with petechiae (Fig. 1A) and tender lymphadenopathy developed in the neck and inguinal regions. Laboratory data showed a white blood cell count of $5.1 \mathrm{~K} / \mu \mathrm{L}$ with a left shift in maturation: metamyelocytes $1 \%$, bands $3 \%$, segs $65 \%$, monocytes $11 \%$, lymphocytes $9 \%$, and basophils $1 \%$. Serum liver enzymes were normal and the serum phenytoin level was $40.8 \mu \mathrm{g} / \mathrm{mL}$ (therapeutic range $10-20 \mu \mathrm{g} / \mathrm{mL}$ ). Other tests were negative, including serology for infection by Mycobacterium tuberculosis, herpes simplex virus, varicella-herpes zoster virus; and autoimmune testing including anti-nuclear antibody, anti-double stranded DNA antibody, rheumatoid factor, anti-SSA/SSB, anti-neutrophil cytoplasmic antibody, anti-Jo-1, anti-ribosomal-P and anti-Scl-70.

A computed tomography (CT) scan revealed enlarged tonsils and bilateral neck lymphadenopathy (Fig. 1B).

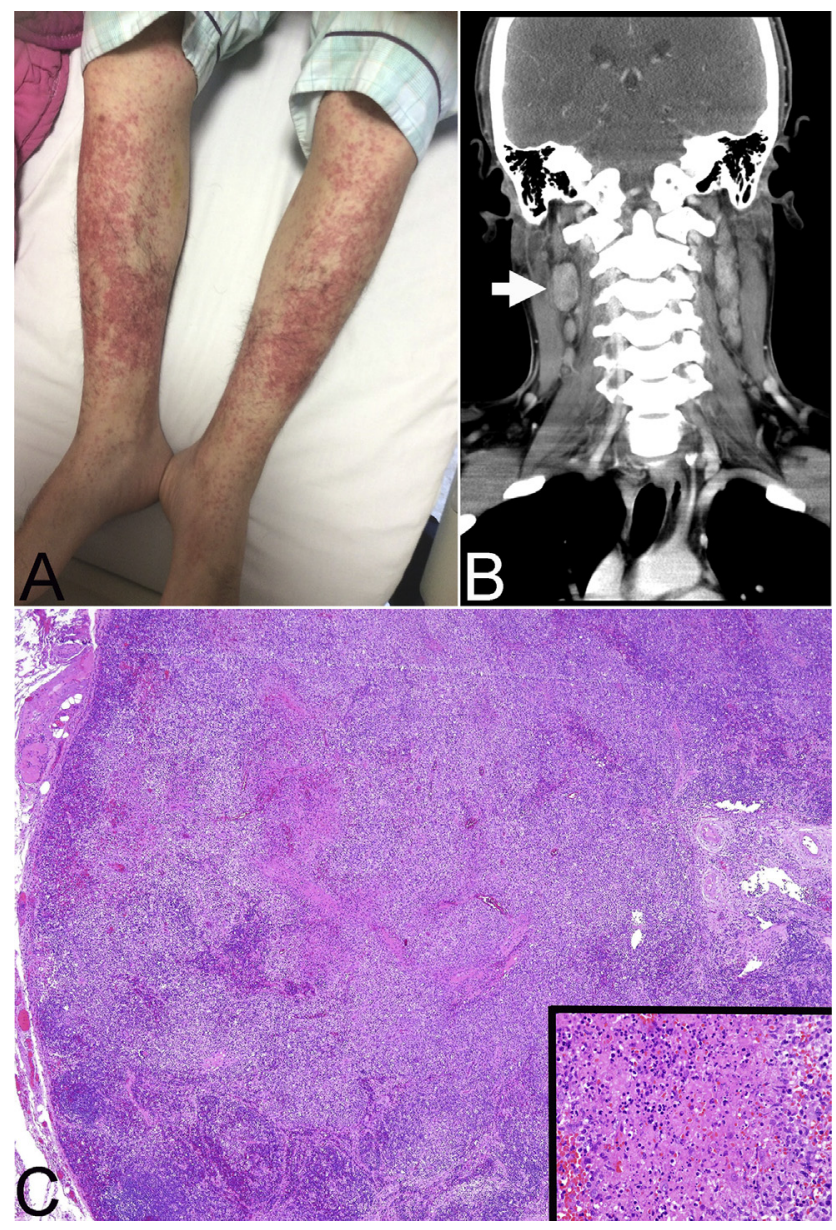

Fig. 1 (A) Generalised maculopapular rashes and multiple petechiae are on bilateral lower extremities. (B) Head and neck computed tomography shows bilateral neck lymphadenopathy, with an enlarged right neck node up to $3 \mathrm{~cm}$ (arrow). (C) Pathologically, the lymph node shows interfollicular expansion containing several necrotising patches composed of karyorrhectic debris and fibrin deposits surrounded by histiocytes (inset, higher magnification). The interfollicular expansion reveals a polymorphic infiltrate.
Phenytoin was stopped on the 14th day after admission and biopsy of a right posterior neck lymph node was performed the next day. The pathological diagnosis was necrotising lymphadenopathy with a differential diagnosis between Kikuchi disease and SLE lymphadenopathy; clinical evaluation was recommended. After withdrawal of phenytoin, fever resolved, skin rash gradually regressed, and lymphadenopathy disappeared within one week. The Naranjo adverse drug reaction probability scale was 8 (Supplementary Fig. 1, Appendix A), indicating a probable relationship between the patient's development of necrotising lymphadenitis and treatment with phenytoin.

Pathologically, the lymph node showed interfollicular (Tzone) expansion with several residual lymphoid follicles. Other fields of interfollicular expansion contained several foci of necrosis composed of karyorrhectic debris and fibrin deposits surrounded by histiocytes (Fig. 1C). The interfollicular expansion revealed a polymorphic infiltrate characterised by small and large lymphocytes, histiocytes and some apoptotic cells indicative of plasmacytoid dendritic cells. Plasma cells and eosinophils were inconspicuous. Immunohistochemical analysis showed that the interfollicular areas were enriched by CD3+ T cells (Fig. 2A,B). CD20 was expressed predominantly by lymphoid follicles (Fig. 2C). Many plasmacytoid dendritic cells in the interfollicular areas were highlighted by CD123 (Fig. 2D). Histiocytes with crescentic (C-shaped) nuclei were positive for myeloperoxidase (Fig. 2E). In situ hybridisation for detection of Epstein-Barr virus was negative (Fig. 2F).

Necrotising lymphadenitis, characterised by focal or geographic necrosis in a lymph node surrounded by an organised cellular response, can be the result of infectious and non-infectious aetiologies. ${ }^{3}$ The differential diagnoses may include Kikuchi-Fujimoto disease, systemic lupus erythematosus, cat-scratch disease, tularaemia, tuberculosis, lymphogranuloma venereum, and viral infections such as herpes simplex virus (HSV) and Epstein-Barr virus. ${ }^{3}$ Neoplastic conditions such as lymphoma and metastatic carcinoma may also contain foci reminiscent of necrotising lymphadenitis. In the current case, there was no granuloma and special studies including acid-fast, GMS stain, HSV immunostain and EBER in situ hybridisation were all negative. Thus, the initial morphological impression was Kikuchi disease and lupus lymphadenitis. After correlation with the clinical history, the morphological features were consistent with phenytoin-induced lymphadenopathy.

Drug reaction with eosinophilia and systemic symptoms (DRESS) syndrome, also known as drug-induced hypersensitivity syndrome (DIHS) or anti-epileptic hypersensitivity syndrome (AHS), is potentially life-threatening, and is characterised by morbilliform skin eruptions, fever, lymphadenopathy, haematological abnormalities, and manifestations of multiorgan dysfunction. ${ }^{6}$ DRESS syndrome is well-known to be caused by phenytoin (phenytoin hypersensitivity syndrome) and other medications, such as allopurinol, antibiotics and nonsteroidal anti-inflammatory drugs (NSAIDs). ${ }^{7}$ DRESS syndrome has a latency interval of 2-6 weeks from drug administration to syndrome onset. The pathogenesis of DRESS syndrome remains to be clarified, but reactivation of human herpes virus 6 (HHV6) with lymphocytic activation may play a role. ${ }^{5,7}$ Withdrawal of the offending agent was the most important part of treatment. Supportive care may be adequate for patients with mild DRESS syndrome. Glucocorticoids, 

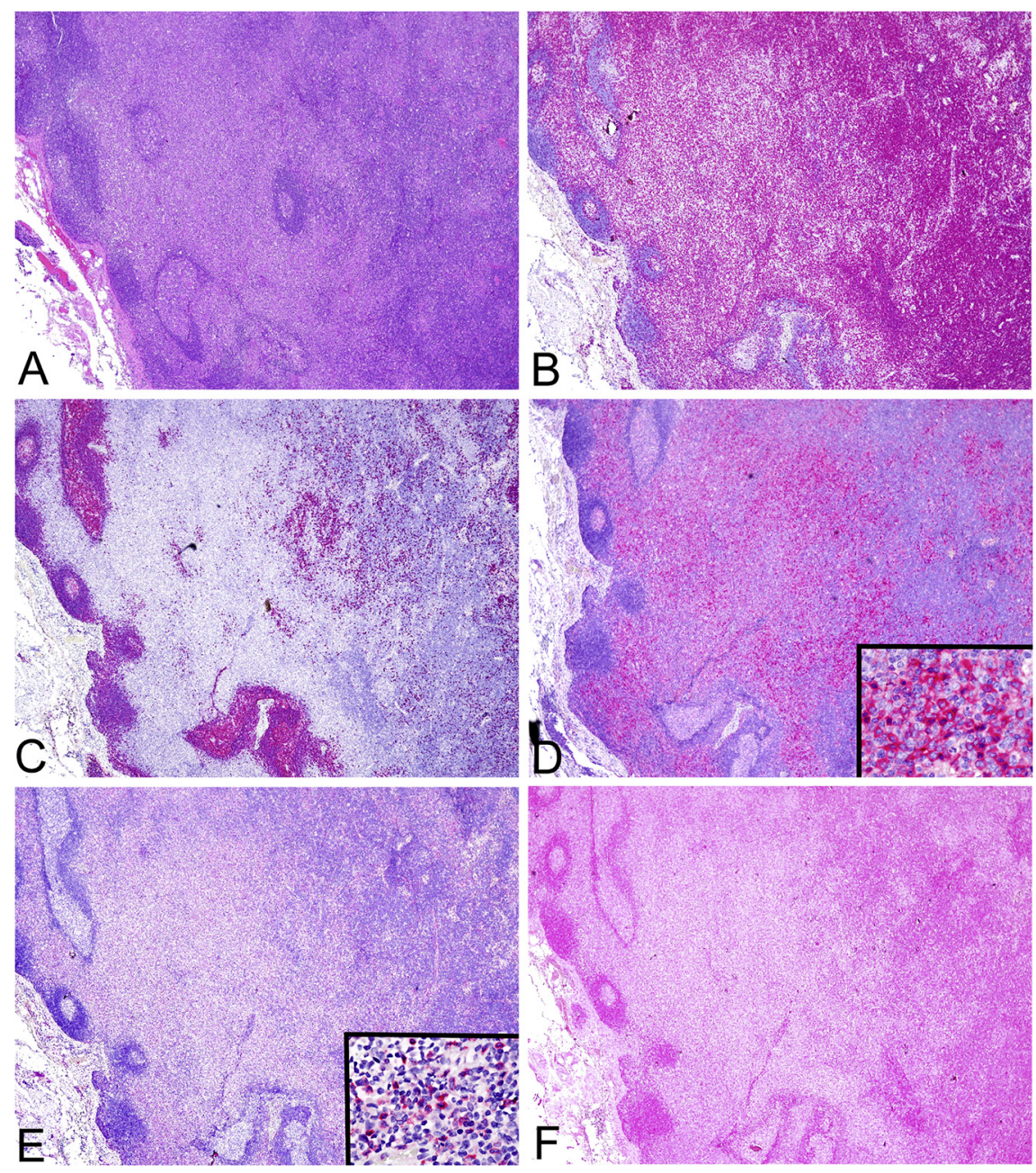

Fig. 2 (A) As shown in the corresponding H\&E section, (B) interfollicular areas are enriched by CD3+ T cells. (C) CD20 is expressed predominantly by lymphoid follicles. (D) Many plasmacytoid dendritic cells in interfollicular areas are highlighted on CD123 stain (inset, high magnification). (E) Histiocytes with crescentic nuclei are positive by myeloperoxidase stain (inset, high magnification). (F) In situ hybridisation for detection of Epstein-Barr virus is negative.

intravenous immunoglobulin (IVIG), plasmapheresis and Nacetylcysteine have been used for severe DRESS cases. ${ }^{8}$

The morphological spectrum of phenytoin (Dilantin)associated lymphadenopathy is broad and may include lymphoid hyperplasia with or without immunoblastic hyperplasia, ${ }^{9}$ granulomatous lymphadenopathy, ${ }^{10}$ immunoblastic lymphadenopathy, ${ }^{11}$ and necrotising lymphadenitis. ${ }^{12}$ Cases of Hodgkin lymphoma and non-Hodgkin lymphoma also have been reported. ${ }^{9}$ The finding of necrotising lymphadenitis is the least frequent and has been reported only once. ${ }^{12}$ In the previous case, the patient presented with a similar clinical picture of fever, lymphadenopathy, and mucocutaneous rashes. Both patients showed no atypical lymphocytosis or eosinophilia. The previously reported patient had prominent liver enzyme elevation. Interestingly, carbamazepine, another anti-epileptic drug may produce lymphadenopathy resembling Kikuchi disease as we reported here. ${ }^{13}$

In conclusion, we report this case to emphasise that phenytoin exposure uncommonly causes necrotising lymphadenitis and should be included in the differential diagnosis of this finding. Withdrawal of the offending agent, in this case phenytoin, was the key to successful management of this patient. This current case also alerts pathologists that drugassociated lymphadenopathy may be occasionally reminiscent of Kikuchi disease.
Conflicts of interest and sources of funding: The authors state that there are no conflicts of interest to disclose.

\section{APPENDIX A. SUPPLEMENTARY DATA}

Supplementary data to this article can be found online at https://doi.org/10.1016/j.pathol.2019.04.010.

\section{Po-Yu Lin', Hui-Wen Chen ${ }^{2}$, L. Jeffrey Medeiros ${ }^{3}$, Kung-Chao Chang ${ }^{2}$}

${ }^{1}$ Department of Neurology, National Cheng Kung University Hospital, College of Medicine, National Cheng Kung University, Tainan, Taiwan; ${ }^{2}$ Department of Pathology, National Cheng Kung University Hospital, College of Medicine, National Cheng Kung University, Tainan, Taiwan; ${ }^{3}$ Department of Hematopathology, The University of Texas MD Anderson Cancer Center, Houston, TX, USA

Contact Kung-Chao Chang, MD, PhD.

E-mail: changkc@mail.ncku.edu.tw

1. Deaver D, Horna $\mathrm{P}$, Cualing $\mathrm{H}$, et al. Pathogenesis, diagnosis, and management of Kikuchi-Fujimoto disease. Cancer Control 2014; 21: $313-21$.

2. Kuo TT. Kikuchi's disease (histiocytic necrotizing lymphadenitis). A clinicopathologic study of 79 cases with an analysis of histologic 
subtypes, immunohistology, and DNA ploidy. Am J Surg Pathol 1995; 19: 798-809.

3. O'Malley DP, George TI, Orazi A, et al. Benign and Reactive Conditions of Lymph Node and Spleen. Atlas of Nontumor Pathology. Washington, DC: American Registry of Pathology, 2009.

4. Chen P, Lin JJ, Lu CS, et al. Carbamazepine-induced toxic effects and HLA-B*1502 screening in Taiwan. N Engl J Med 2011; 364: 1126-33.

5. Fleming P, Marik PE. The DRESS syndrome: the great clinical mimicker. Pharmacotherapy 2011;31: 332

6. De A, Rajagopalan M, Sarda A, et al. Drug reaction with eosinophilia and systemic symptoms: an update and review of recent literature. Indian J Dermatol 2018; 63: 30-40.

7. Husain Z, Reddy BY, Schwartz RA. DRESS syndrome: Part I. Clinical perspectives. J Am Acad Dermatol 2013; 68. 693e1-14; 706-8.

8. Cumbo-Nacheli G, Weinberger J, Alkhalil M, et al. Anticonvulsant hypersensitivity syndrome: is there a role for immunomodulation? Epilepsia 2008; 49: 2108-12.

9. Abbondazo SL, Irey NS, Frizzera G. Dilantin-associated lymphade nopathy. Spectrum of histopathologic patterns. Am J Surg Pathol 1995 19: $675-86$.

10. Ovallath S, Remya RK, Kumar C, et al. Granulomatous lymphadenopathy secondary to phenytoin therapy. Seizure 2013; 22: 240-1.

11. Lapes MJ, Vivacqua RJ, Antoniades K. Immunoblactic lymphadenop athy associated with phenytoin (diphenylhydantoin). Lancet 1976; 1 : 198.

12. Subbannan K, Gujral JS. Necrotizing lymphadenitis associated with the phenytoin-induced hypersensitivity syndrome. South Med J 2005; 98: 937-9.

13. Ganga A, Corda D, Gallo Carrabba G, et al. A case of carbamazepineinduced lymphadenopathy resembling Kikuchi disease. Eur Neurol 1998: 39: 247-8.

DOI: https://doi.org/10.1016/j.pathol.2019.04.010

\section{Is secretory breast carcinoma underdiagnosed? In the era of targeted therapy should there be a low threshold to screen for NTRK immunohistochemistry in triple negative breast cancers?}

Sir,

Secretory breast carcinomas are reported to be very rare, accounting for $<0.1 \%$ cases of invasive breast cancers, but may be under-recognised. ${ }^{1}$ Initially termed juvenile breast carcinoma', ${ }^{2}$ secretory breast carcinomas are characteristically driven by oncogenic ETV6-NTRK3 gene fusions which are extremely rare in all other breast carcinomas. ${ }^{1}$ The neurotrophic tyrosine receptor kinase (NTRK) group of genes, NTRK1, NTRK2 and NTRK3, encode for a family of receptor tyrosine kinases $\operatorname{Trk} \mathrm{A}, \operatorname{TrkB}$, and $\operatorname{TrkC}$, respectively. ${ }^{3,4}$ These receptors normally contribute to neuronal development, function, survival and proliferation. ${ }^{3,4}$ In addition to secretory carcinoma of the breast, uncontrolled activation of the NTRK genes, usually due to fusion events, have proven to be the most common driver of some relatively rare tumours (including infantile fibrosarcoma and secretory carcinoma of the salivary gland) but are also found at a very low incidence in a diverse range of malignancies. ${ }^{3-5}$

$N T R K$ gene fusions are becoming increasingly important to identify in the routine clinical laboratory because of the availability of rationally designed targeted therapies including the specific NTRK inhibitor larotrectinib. ${ }^{3-5}$ Larotrectinib has demonstrated efficacy against a wide variety of tumours harbouring activating NTRK fusions and is now US Food and Drug Administration approved.
However, the optimal strategy for identifying NTRK fusions in the routine clinical setting is still unclear. While screening immunohistochemistry has been suggested to have a potential role in triaging molecular testing, it is not completely sensitive or specific and expensive molecular methods remain the gold standard. ${ }^{6}$ Whilst it would be beyond the resources of most pathology laboratories to screen all malignancies with a low risk for NTRK fusions (for example all breast carcinomas) with molecular techniques, it may be reasonable to justify the cost for specific tumours with a high incidence of NTRK fusions (for example secretory carcinomas or infantile fibrosarcomas). Despite questions about its sensitivity and specificity, it may be reasonable to perform screening NTRK immunohistochemistry to triage molecular testing on tumours with a low or intermediate risk of harbouring NTRK fusions.

Typically secretory breast carcinomas lack oestrogen receptor, progesterone receptor and human epidermal growth factor (HER2) expression, and therefore can be considered a specific example of triple negative breast cancer (TNBC). ${ }^{4}$ That is, the subgroup of all breast carcinomas classified as TNBC is enriched for secretory carcinoma and may have a higher incidence of NTRK gene rearrangements, particularly if secretory carcinoma is under-recognised. Therefore, we postulated that there may be a role for NTRK immunohistochemistry in all TNBCs to triage molecular testing. Indeed there are case reports of tumours initially thought to be TNBC being re-classified as secretory carcinoma upon review and this having a significant impact on treatment.

We sought to assess whether screening immunohistochemistry for NTRK may have a role in the routine assessment of TNBC in order to triage molecular testing, by performing NTRK immunohistochemistry in a large number of TNBCs with follow up molecular testing on all cases with some positive staining.

A tissue microarray (TMA) of consecutive primary triple negative breast carcinomas was used. The TMA included two $1 \mathrm{~mm}$ cores from all triple negative breast carcinomas that had undergone resection between 2005 and 2015 at our institution. The clinical characteristics and androgen receptor status of this cohort have been previously reported. ${ }^{8} \mathrm{We}$ performed pan-NTRK immunohistochemistry using a commercially available rabbit monoclonal antibody (clone EPR17341; Abcam, USA; dilution 1/50, after heat induced antigen retrieval in an alkaline retrieval solution) using similar methods to that reported by others.

The TMA sections stained with NTRK were scored by two independent observers who were blinded to all clinical and pathological details. NTRK IHC was repeated on whole sections from all cases with any positive staining identified by one or both observers.

There were 137 cases in this TMA cohort, of which 11 (8\%) demonstrated some positive staining on TMAs. All had at least some confirmed positive expression when the scoring was repeated on whole sections. The tumour and patient characteristics of the 11 positive cases are presented in Table 1. Briefly, the ages ranged from 45 to 83 years (mean 59.5). There was one case initially diagnosed as secretory breast carcinoma (the only secretory carcinoma diagnosed at our institution over the 11 year period). Eight cases were originally classified as invasive ductal carcinomas of no special type and two as invasive ductal carcinomas with medullary features. 